\title{
Interpositional dural graft technique for the treatment of dural arteriovenous fistulas
}

\author{
Michael Finn, M.D., Paul Klimo JR., M.D., M.P.H., \\ and William T. Couldwell, M.D., Ph.D. \\ Department of Neurosurgery, University of Utah, Salt Lake City, Utah
}

\begin{abstract}
$\checkmark$ Dural arteriovenous fistulas (dAVFs) are acquired direct arteriovenous shunts that often drain into the dural venous sinus. Treatment options generally involve disrupting the abnormal vascular conduits by using a combination of modalities, including surgical disconnection, radiosurgery, and transarterial and transvenous embolization. Often these modalities provide only partial treatment of fistulous lesions, and thus the fistula recurs and symptoms result. The authors report on a novel surgical technique in which the involved venous sinuses are skeletonized and an interpositional dural substitute is placed between the disconnected sinus and native dura mater and over the pial surface adjacent to the sinus. The technique, which is demonstrated in an illustrative case, is intended to preserve native venous drainage and to prevent recruitment of new vascularization to the venous sinus postoperatively. The authors have not observed reconstitution of fistulas over areas treated with this technique, which offers the advantage of inhibiting vascular ingrowth (refistulization) while maintaining venous sinus patency.
\end{abstract}

KEY WoRdS • dural arteriovenous fistula • dural substitute • graft

$\mathrm{D}$ URAL arteriovenous fistulas are direct AV shunts located within the dura mater; they account for 10 to $15 \%$ of all intracranial AV shunts. ${ }^{5,11}$ These lesions have been reported to occur in close association with dural sinus thrombosis, intracranial infection, trauma, surgery, surgical occlusion of the dural sinuses, pregnancy, and hypercoagulable states. ${ }^{16}$ Theories about the origin of these lesions center on the creation of venous hypertension caused by venous outflow obstruction. ${ }^{7}$ Increased venous pressure may then either open preexisting AV communications within the dura mater ${ }^{3,14}$ or stimulate the formation of new vessels due to the release of angiogenic factors in response to the local ischemia. ${ }^{7} \mathrm{~A}$ vicious cycle can occur in which increased hypertension leads to the further recruitment of preexisting fistulas or the creation of new shunts, which can in turn lead to further hypertension.

Current treatment modalities focus on the disruption of abnormal AV communications or resection of the involved sinus if feasible. Both endovascular and open surgical options exist, and these can be used alone or in combination. ${ }^{2}$ Radiosurgery is a treatment option that is also being used with increased frequency. ${ }^{12}$ Although good control of symptoms has been reported using currently

Abbreviations used in this paper: $\mathrm{AV}=$ arteriovenous; $\mathrm{dAVF}=$ dural arteriovenous fistula. available modalities, ${ }^{2}$ the risk of treatment failure is high, with failure rates of 11 to $100 \%$, depending on the treatment modality used and the location and aggressiveness of the lesion. ${ }^{10}$

We here report on a novel technique in which an interpositional modified xenograft dural patch graft is placed between the sinus and the native dura mater after the skeletonization of sinuses. A graft is also placed over the pial surface to prevent fistulization. We believe that this technique might reduce the risk of recurrent dAVFs in the future by preserving cerebral venous drainage and thus reducing venous hypertension that promotes fistula development, and by introducing a physical barrier that may prevent recruitment of new vasculature.

\section{Surgical Procedure}

The goal of the operation is to physically interrupt arterial channels within the dura mater that directly enter the involved sinus(es). The initial dural incision is made approximately 2 to $3 \mathrm{~mm}$ from the edge of the sinus and is carried parallel to the sinus. Brisk bleeding from the cut dural edge can again occur over the length of the affected sinus and can be controlled with bipolar electrocautery or hemostatic clips, if necessary. A piece of dura (bovine pericardium cross-linked with glutaraldehyde, DuraGuard dural repair patch, Synovis) is tailored to fit between the 


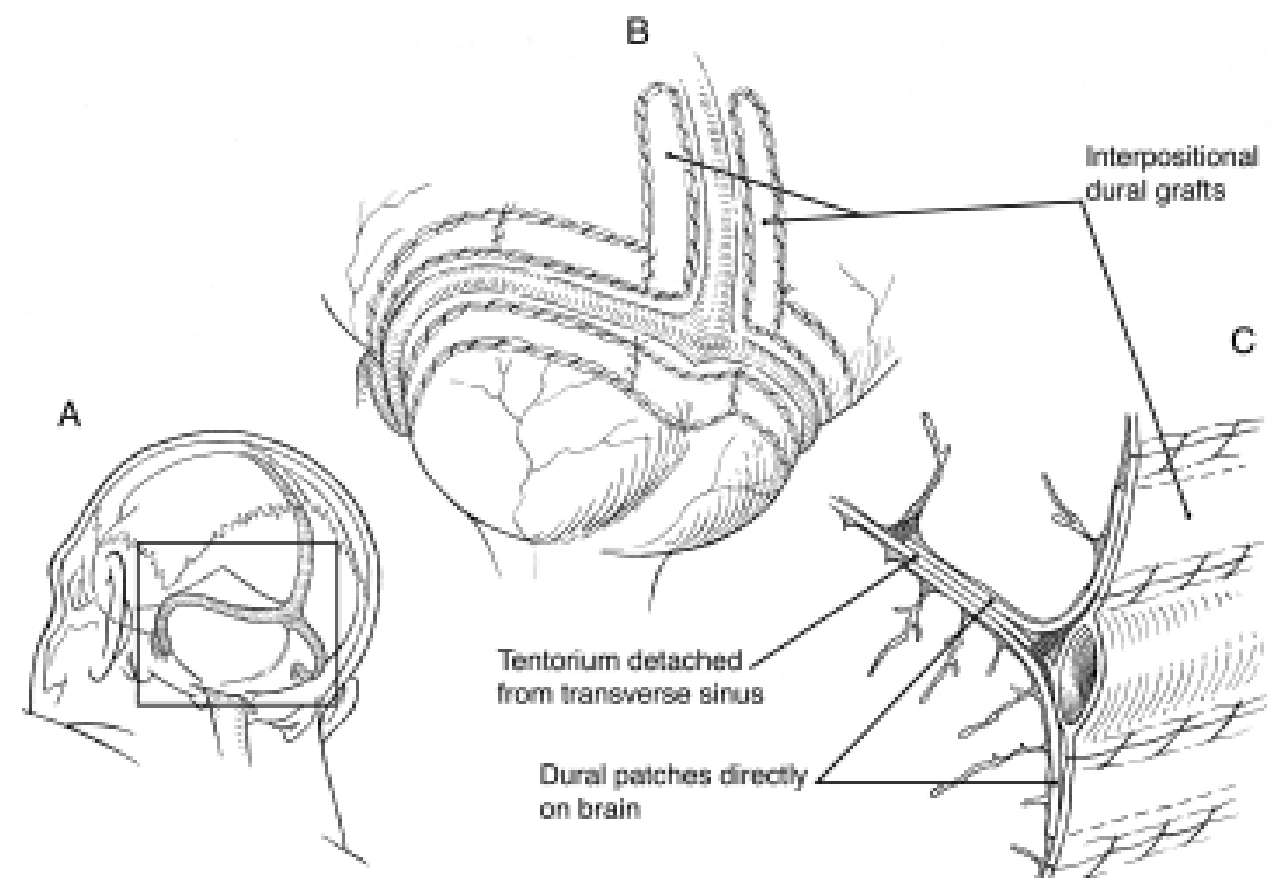

FIG. 1. Drawings depicting placement of a dural graft. A: The interpositional dural graft is placed along the borders of the sagittal sinus and the bilateral sigmoid/transverse sinuses. B: The interpositional xenograft is placed between the sinus and the disconnected dura. C: Note direct placement of the xenograft on the cerebral and cerebellar surfaces to prevent direct refistulization from the pial surface.

cut edges of the native dura as demonstrated in Fig. 1. As opposed to other newer synthetic substitutes that eventually allow the ingrowth of native tissue, we prefer DuraGuard because it is inert and essentially forms a barrier against the vascular ingrowth. Disconnecting the sigmoid sinus, especially the presigmoid dura, can be challenging but is achieved with adequate drilling of the surrounding bone. Disconnecting the transverse sinus from the tentorium is imperative, but suturing an interpositional graft is more difficult because of the physical constraints in this region. Finally, a piece of dural repair patch is placed on the brain itself to physically separate it from the sinus if there is concern about the development of pial fistulas.

\section{Illustrative Case}

History and Examination. This 28-year-old man presented with symptoms of intracranial hypertension, including constricted visual fields requiring optic nerve sheath fenestration, and several weeks of increasing audible bruit. One year earlier, he had sustained a transverse sinus thrombosis after an episode of dehydration, which was treated with anticoagulation therapy. His examination was remarkable for an audible bruit over the left temporal and suboccipital area and palpable feeding vessels over the suboccipital region. Results of an angiographic evaluation revealed an extensive dAVF involving the torcular herophili, the bilateral transverse sinuses, and the left sigmoid sinus. Feeding arteries emanated from the vertebral, occipital, auricular, middle meningeal, tentorial, and superficial temporal arteries. The left sigmoid-jugular junction was noted to be mildly stenotic, and retrograde flow from the left sigmoid and transverse sinus to the right transverse sinus was present. Cortical reflux was also noted (Fig. 2).

Treatment. The patient underwent embolization of the multiple feeding arteries, including those from the left occipital, left middle meningeal, and left superficial temporal arteries. The procedure resulted in marked improvement in venous flow, with resumption of antegrade flow in the left sigmoid and transverse sinuses, but feeding arteries from the posterior meningeal branch of the left vertebral artery and the left tentorial artery were still present.

Two days after embolization, the patient underwent surgical skeletonization of the bilateral transverse sinuses and posterior sagittal sinus with placement of an interpositional dural graft. Specifically, the patient's transverse sinus on the left was skeletonized to the transverse-sigmoid junction superiorly, along the sigmoid to the jugular bulb inferiorly, and along the tentorium from the torcular herophili to the transverse-sigmoid junction. On the right side, skeletonization was performed to the transverse-sigmoid junction both superiorly and inferiorly. A postoperative angiogram demonstrated no remaining fistula over the region of disconnection (Fig. 3).

Posttreatment Course. The patient has been followed up for a period of 3 years with no evidence of new fistulization at the disconnected sites.

Note that in our experience with six patients, no demonstrable recurrence of fistulization has been observed over the region of inert graft placement (range of follow up 1 month -4 years).

\section{Discussion}

We described a modification to Lucas and colleagues" ${ }^{9}$ technique of sinus skeletonization for the surgical treat- 


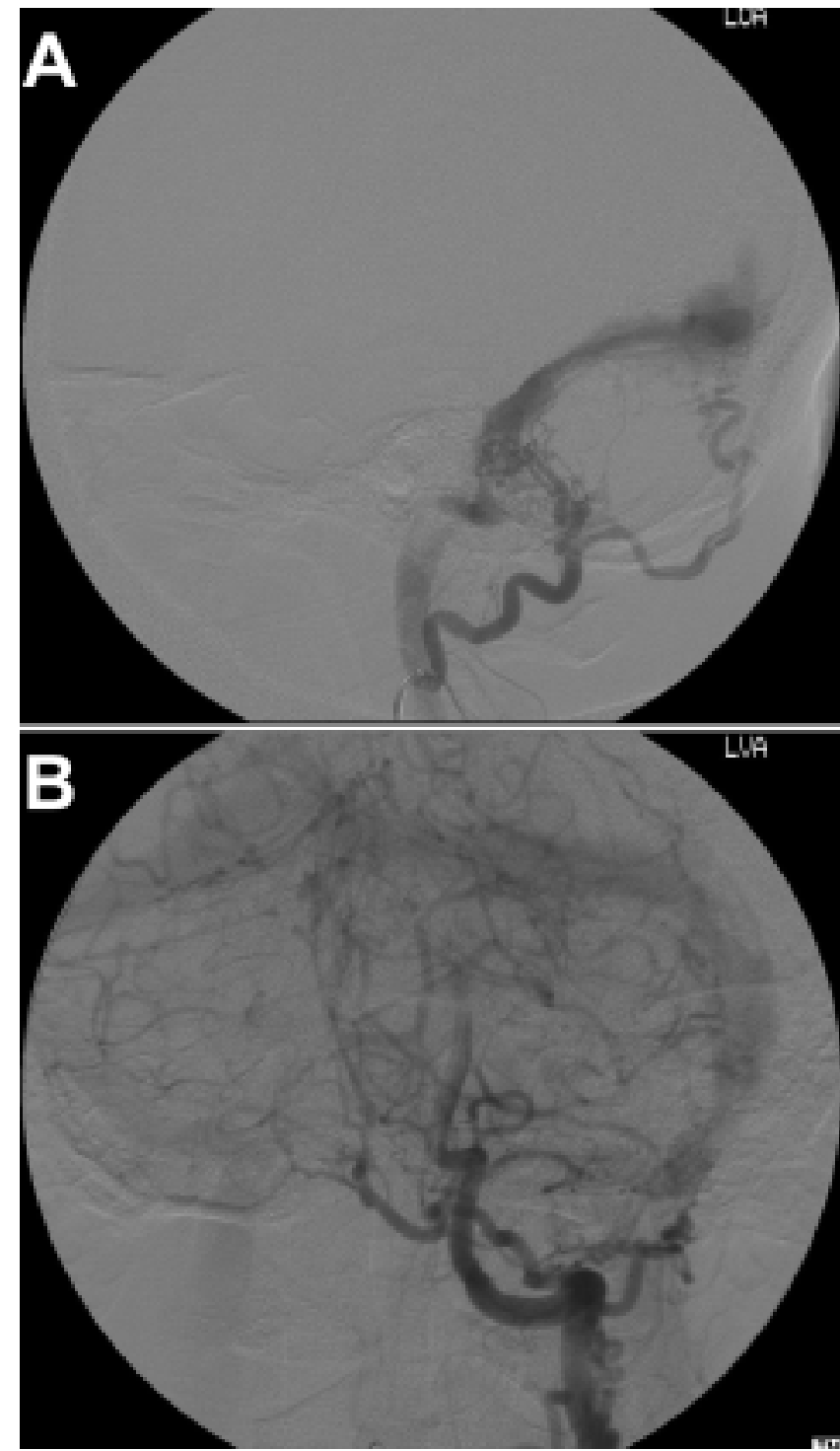

FIG. 2. A: Left occipital artery angiogram demonstrating a prominent occipital artery with multiple retroauricular branches fistulizing to the sigmoid sinus. B: Left vertebral artery angiogram showing prominent posterior meningeal and muscular branches supplying the fistula.

ment of dAVFs with sinus drainage. This modification can be useful in preventing the recurrence of fistulas by imposing a mechanical barrier between native dura mater and the sinus while maintaining the patency of the involved sinuses. We demonstrated this procedure in a patient who tolerated it without surgical complication or recurrence of the fistula.

Current open surgical and endovascular treatments of dAVFs are focused on eradicating one (arterial or venous) or both sides of the fistula. Specifically, this process involves physically disconnecting the feeding arteries or obliterating or resecting the involved sinuses. Endovascular arterial embolization rarely results in permanent obliteration of fistulas and is now mostly used for palliation and as a preoperative aid. ${ }^{1,10}$ Sinus obliteration via a transvenous endovascular approach or combined with an open surgical approach has been proposed;, ${ }^{4}, 15$ however, its use has been tempered because it is only appropriate in cases in which the sinus is arterialized, it fails to maintain or restore normal venous drainage, and it can have significant complications including venous infarction and hemorrhage. ${ }^{2}$ Open resection of the affected sinus has also been performed..$^{13}$ Additionally, only directly arterialized sinuses can be resected with adequate collateral venous drainage. The boundaries of resectable and unresectable sinuses can be difficult to ascertain intraoperatively, and venous infarction can result with the obliteration of normal sinus drainage. Radiosurgical obliteration of dAVFs is being used as a surgical adjunctive or primary treatment with some success. Although cure rates of up to $72 \%$ have
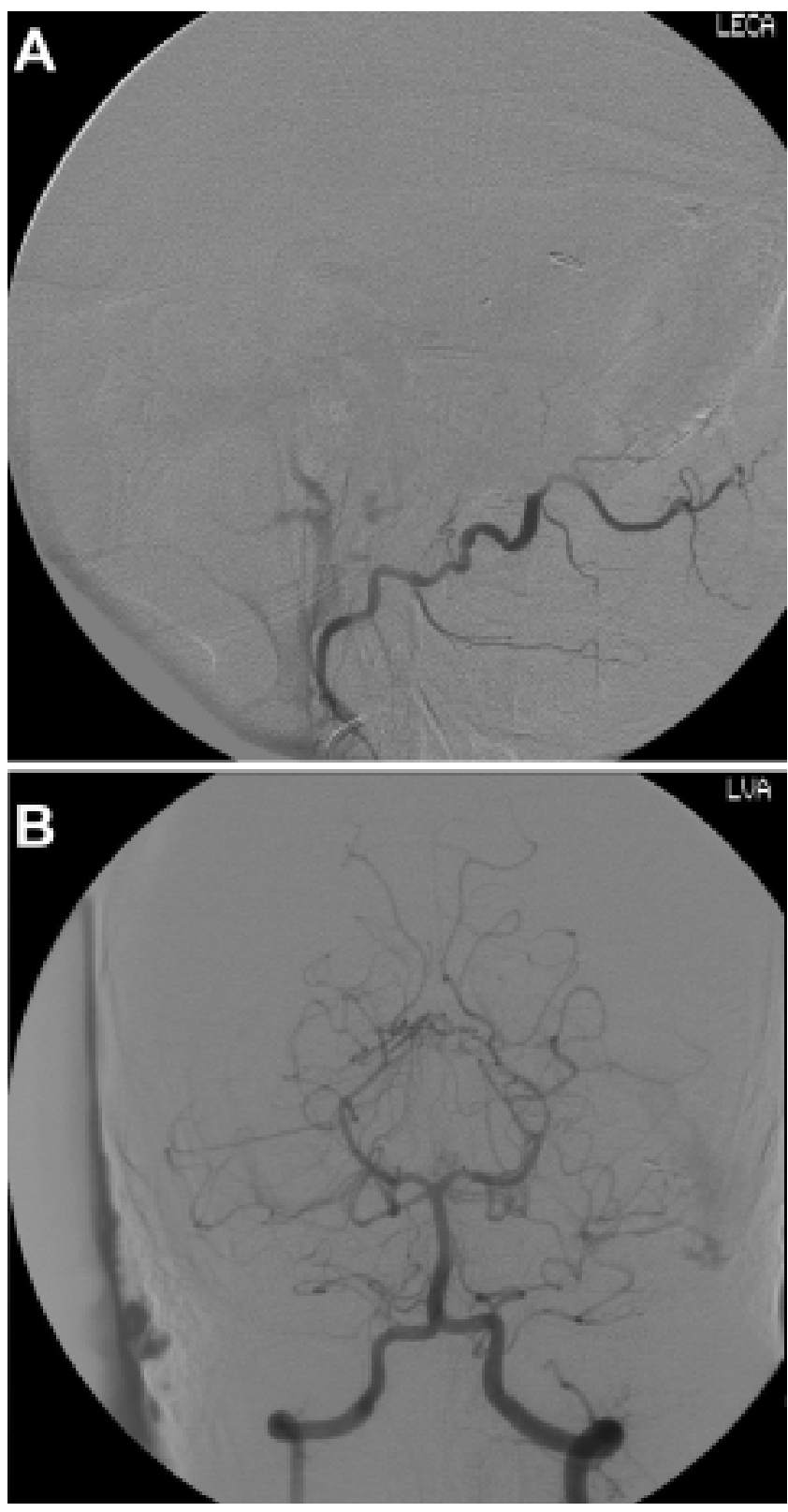

FIG. 3. Left external carotid artery (A) and vertebral artery (B) angiograms revealing absence of fistulous communication over the disconnected region postoperatively. 
been reported, obliteration can take up to 2 years, during which time the patient is still at risk of hemorrhage. . $^{8,12}$ Finally, surgical skeletonization of the dural sinuses has been described as an effective tool in dealing with this disease process. ${ }^{69}$ However, simple interruption of the dura mater without placement of an interpositional graft may not provide an adequate barrier for revascularization, which has been witnessed in previous cases treated by the authors and others.

\section{Conclusions}

We reported on a technique for the treatment of dAVFs in which the affected sinuses are skeletonized and isolated with the placement of a dural interpositional graft. Thus far we have not observed reconstitution of fistulas over areas treated using this technique. The procedure offers the advantage of maintaining venous sinus patency, which may reduce the development of further venous hypertension and thereby decrease the pathophysiological impetus for the development of additional fistulas.

\section{References}

1. Awad IA: Intracranial dural arteriovenous malformations, in Wilkins RH, Rengachary S (eds): Neurosurgery. New York: McGraw-Hill, 1996, pp 2519-2527

2. Collice M, D'Aliberti G, Arena O, Solaini C, Fontana RA, Talamonti G: Surgical treatment of intracranial dural arteriovenous fistulae: role of venous drainage. Neurosurgery 47: $56-67,2000$

3. Graeb DA, Dolman CL: Radiological and pathological aspects of dural arteriovenous fistulas. Case report. J Neurosurg 64: 962-967, 1986

4. Halbach VV, Higashida RT, Hieshima GB, Wilson CB, Hardin $\mathrm{CW}$, Kwan E: Treatment of dural fistulas involving the deep cerebral venous system. AJNR Am J Neuroradiol 10: 393-399, 1989

5. Hamada Y, Goto K, Inoue T, Iwaki T, Matsuno H, Suzuki S, et al: Histopathological aspects of dural arteriovenous fistulas in the transverse-sigmoid sinus region in nine patients. Neurosurgery 40:452-458, 1997
6. Hugosson R, Bergstrom K: Surgical treatment of dural arteriovenous malformation in the region of the sigmoid sinus. $\mathbf{J}$ Neurol Neurosurg Psychiatry 37:97-101, 1974

7. Lawton MT, Jacobowitz R, Spetzler RF: Redefined role of angiogenesis in the pathogenesis of dural arteriovenous malformations. J Neurosurg 87:267-274, 1997

8. Link MJ, Coffey RJ, Nichols DA, Gorman DA: The role of radiosurgery and particulate embolization in the treatment of dural arteriovenous fistulas. J Neurosurg 84:804-809, 1996

9. Lucas CP, De Oliveira E, Tedeschi H, Siqueira M, Lourenzi M, Piske RL, et al: Sinus skeletonization: a treatment for dural arteriovenous malformations of the tentorial apex. Report of two cases. J Neurosurg 84:514-517, 1996

10. Lucas CP, Zabramski JM, Spetzler RF, Jacobowitz R: Treatment for intracranial dural arteriovenous malformations: a meta-analysis from the English language literature. Neurosurgery 40:1119-1132, 1997

11. Newton TH, Weidner W, Greitz T: Dural arteriovenous malformation in the posterior fossa. Radiology 90:27-35, 1968

12. Söderman M, Edner G, Ericson K, Karlsson B, Rähn T, Ulfarsson E, et al: Gamma knife surgery for dural arteriovenous shunts: 25 years of experience. J Neurosurg 104:867-875, 2006

13. Sundt TM Jr, Piepgras DG: The surgical approach to arteriovenous malformations of the lateral and sigmoid dural sinuses. J Neurosurg 59:32-39, 1983

14. Terada T, Higashida RT, Halbach VV, Dowd CF, Tsuura M, Komai N, et al: Development of acquired arteriovenous fistulas in rats due to venous hypertension. J Neurosurg 80:884-889, 1994

15. Urtasun F, Biondi A, Casaco A, Houdart E, Caputo N, Aymard A, et al: Cerebral dural arteriovenous fistulas: percutaneous transvenous embolization. Radiology 199:209-217, 1996

16. Vilela P, Willinsky R, terBrugge K: Dural arteriovenous fistula associated with neoplastic dural sinus thrombosis: two cases. Neuroradiology 43:816-820, 2001

Manuscript submitted January 15, 2007.

Accepted January 26, 2007.

Current address for Dr. Klimo: Wright-Patterson Air Force Base, Ohio.

Address reprint requests to: William T. Couldwell, M.D., Ph.D., Department of Neurosurgery, University of Utah School of Medicine, 175 N Medical Drive, Salt Lake City, Utah 84132. email: william.couldwell@hsc.utah.edu. 